\section{EFFECT OF HOSPITALISATION ON AMBULATORY BLOOD PRESSURE MONITORING VALUES IN CHILDREN 10 TO 16 YEARS OF AGE}

Ines Vidatic* ${ }^{*}$ Slavica Bošnjak, Bernardica Valent Morić. University Hospital Centre Sestre milosrdnice, Zagreb

\subsection{6/archdischild-2021-europaediatrics.379}

Aim Ambulatory blood pressure monitoring (ABPM) is a standard procedure in diagnosing arterial hypertension in children and adolescents. ABPM is usually used as an outpatient method enabling recordings of blood pressure (BP) during regular daily activities. However, sometimes it is necessary to have an insight into 24-hour BP values during hospitalisation which can result in clinical doubt of validity of those measurements since hospitalisation restricts physical activity in children providing falsely lower results. On the other hand, hospitalisation can be stressful to children, resulting in values higher than those measured outside of the hospital environment. The aim of this study was to analyze the effect of hospitalization on ambulatory blood pressure (ABP) values in children.

Methods The research was conducted at the Department of Pediatrics, University Hospital Centre Sestre milosrdnice. The study included 72 children of both sexes, aged 10 and 16 years. In our study group $40(55.6 \%)$ of children had arterial hypertension, $9(12.5 \%)$ had type 1 diabetes while the diagnosis in $23(31.9 \%)$ of subjects was headache, precordial pain, overweight or obesity, asthma or proteniuria. ABPM was performed on all subjects. The first group included children to whom ABPM was performed in the hospital, while in the second group ABPM was done as an outpatient procedure. Height, weight and body mass index were obtained for all subjects. Office BP, standard ABPM parameters and blood pressure variability (BPV) were analysed.

Results There was no statistically significant difference between hospitalised and outpatient ABP results for 24-hour systolic (SBP) and diastolic blood pressure (DBP) $(\mathrm{p}=0.173$ and $\mathrm{p}=0.93$, respectively), daytime SBP and DBP $(\mathrm{p}=0.268$ and $p=0.968$, respectively) and nighttime SBP and DBP $(p=0.116$ and $\mathrm{p}=0.653$, respectively). Also, there was no difference neither for systolic $(p=0.921)$ and diastolic $(p=0.238)$ BP load nor for the nighttime dipping $(\mathrm{p}=0.181$ for systolic dipp and $\mathrm{p}=0.479$ for diastolic dipp). 24-hour and daytime diastolic BPV was, however, higher in an outpatient subjects $(p=0.034$ and $\mathrm{p}=0.001$, respectively). Most of the subjects had non-dipping pattern $(52.78 \%$ in hospitalised subjects and in $66.67 \%$ in outpatient subjects).

Conclusion Hospitalization did not affect the standard ABPM parameters. Higher diastolic BPV in outpatient children could indicate somewhat higher physical activity in this group, but without affecting the average BP values. These results indicate a low level of physical activity in our subjects.

\section{WHAT INFLUENCES BLOOD PRESSURE VARIABILITY IN CHILDREN WITH ESSENTIAL HYPERTENSION?}

\footnotetext{
${ }^{1}$ Iva Škorić* ${ }^{1}$ Ivana Trutin, ${ }^{2,3}$ Karolina Kramarić, ${ }^{2}$ Ivica Škoro, ${ }^{2,3,4}$ Matej Šapina ${ }^{1}$ Department of Pediatrics, Sestre milosrdnice University Hospital Centre, Zagreb, Croatia ${ }^{2}$ University Hospital Osijek, Osijek, Croatia; ${ }^{3}$ Faculty of Dental Medicine and Health, J Strossmayer University of Osijek; ${ }^{4}$ Faculty of Medicine, JJ Strossmayer University of Osijek, Osijek, Croatia
}

Blood pressure variability (BPV) is a complex physiological phenomenon and it is known that increased BPV exacerbates the progression of hypertension and correlates with adverse cardiovascular outcomes. However, there is paucity of data regarding factors influencing BPV in pediatric population. This study aimed to analyze blood pressure (BP) pattern and assess blood pressure variability and its possible determinants in untreated children with essential hypertension.

The study group included 132 children, average age 15 y (7- 18 y), 84 boys and 48 girls with essential hypertension and no use of antihypertensive therapy. Anthropometric parameters, serum glucose level (sG), serum uric acid (sUA), serum creatinine (sCr), total cholesterol (TCh), HDL cholesterol (HDL), LDL cholesterol (LDL) and triglycerides (TG) were measured in all participants. Office blood pressure (BP) and ambulatory blood pressure (AMBP) was measured according to recent European Society of Hypertension (ESH) guidelines. BPV was defined as value of the standard deviation of blood pressure for day and night time periods. In addition to the classical statistical analysis, an unsupervised machine learning approach using the expected maximization algorithm was implemented to find groups of patients with similar characteristics.

The average age was $14.98 \pm 2.1$ years. Comparing sexspecific BP parameters, boys had higher daytime and nighttime systolic BP, although the difference was not statistically significant $(p=0.067$ and $p=0.583)$. Girls had significantly higher daytime and nighttime diastolic BP values $(p=0.017$; $\mathrm{p}=0.027)$. There was no difference in BPV according to gender while boys have higher values of sCr, sG, and sUA despite similar body mass index (BMI) $[27.18 \pm 5.27 \mathrm{~kg} / \mathrm{m} 2$ (males) vs $27.94 \pm 5.27 \mathrm{~kg} / \mathrm{m} 2$ (females), $\mathrm{p}=0.427]$. A statistically significant positive correlation was found between the Z-score for BMI and daytime systolic BPV, nighttime systolic BP and total cholesterol, nighttime diastolic BPV and sUA $(r=0.19, r=$ 0.28 and $\mathrm{r}=0.26$, respectively). Within the male population two clusters were found. The subjects in cluster 2 had higher daytime and nighttime systolic and diastolic BP values, higher total cholesterol and triglycerides and significantly higher night time systolic and diastolic BPV.

Understanding potential determinants of BPV in hypertensive children could help identify patients with increased cardiovascular risk earlier. Our results suggest that clustering of metabolic factors influence BPV in untreated children with essential hypertension which could also be gender specific for male subjects.

\section{CONTRAST-ENHANCED UROSONOGRAPHY IN DIAGNOSTICS OF INTRARENAL REFLUX}

\begin{abstract}
${ }^{1,2}$ Andrea Cvitković Roić* ${ }^{1}$ Iva Palčić, ${ }^{3}$ Goran Roić. ${ }^{1}$ Helena Clinic for Pediatric Medicine, Kneza Branimira 71, Zagreb, Croatia; ${ }^{2}$ Faculty of Medicine, Josip Juraj Strossmayer University of Osijek, Josipa Huttlera 4, 31000 Osijek, Croatia; ${ }^{3}$ Children's Hospital Zagreb, Ul. Vjekoslava Klaića 16, Zagreb, Croatia
\end{abstract}

10.1136/archdischild-2021-europaediatrics.381

Many studies have shown that intrarenal reflux (IRR) is one of the most important risk factors for febrile urinary tract infections (UTI) and renal scarring and consequently the development of hypertension and renal failure. The incidence of IRR diagnosed by fluoroscopic voiding cystourethrography (VCUG) ranges from below $1 \%$ to $10 \%$. Although numerous 